\title{
LA FAMILIA COMO UNIDAD DE ANÁLISIS EN LA INVESTIGACIÓN CIENTÍFICA EN MEDICINA FAMILIAR
}

\section{THE FAMILY AS A UNIT OF ANALYSIS IN SCIENTIFIC RESEARCH IN FAMILY MEDICINE}

Enrique Villarreal-Ríos ${ }^{1 *}$, Verónica Escorcia-Reyes ${ }^{2}$, Emma R. Vargas-Daza' ${ }^{1}$ Laura A. Cu-Flores³ Lilian Galicia-Rodríguez y Erasto Carballo-Sanander ${ }^{4}$

'Unidad de Investigación Epidemiológica y en Servicios de Salud, Instituto Mexicano del Seguro Social, Santiago de Querétaro; ${ }^{2}$ Coordinación Clínica de Educación e Investigación en Salud, Unidad de Medicina Familiar 6, Instituto Mexicano del Seguro Social, San Juan del Río; ${ }^{3}$ Consulta de Medicina Familiar, Unidad de Medicina Familiar 16, Instituto Mexicano del Seguro Social, Santiago de Querétaro; ${ }^{4}$ Coordinación Clínica de Educación e Investigación en Salud, Unidad de Medicina Familiar 7, Instituto Mexicano del Seguro Social, San Juan del Río. Querétaro, México

RESUMEN: Introducción: En el proceso de investigación, identificar la unidad de análisis es trascendental, en torno a la unidad de análisis se construye la propuesta conceptual y metodológica, incluso la descripción e interpretación de los resultados se realiza en torno a ella y aún más, la discusión del documento. Cuando se realiza investigación en medicina familiar la unidad de análisis es la familia. Objetivo: Realizar una propuesta en torno a la familia como unidad de análisis cuando se realiza investigación científica en medicina familiar. Discusión: En el contexto de la investigación científica, lo aquí planteado tendrá que ser sometido a escrutinio y criticado; aunado a ello, requerirá el desarrollo del marco conceptual que la sostenga y la creación de una metodología científica específica que le otorgue la característica de investigación en medicina familiar. Conclusión: Se propone a la familia como unidad de análisis en la investigación científica de la medicina familiar y como la principal unidad de atención en la asistencia médica de esta especialidad.

Palabras clave: Medicina Familiar. Investigación. Análisis. Servicios de salud.
ABSTRACT: Background: In the research process, identifying the unit of analysis is transcendental, around the unit of analysis the conceptual and methodological proposal is built, even the description and interpretation of the results is carried out around it and even more, the discussion of the document. When research is carried out in family medicine, the unit of analysis is the family. Objective: Make a proposal around the Family as a unit of analysis when scientific research is carried out in Family Medicine. Discussion: The proposed here will have to be analyzed and criticized, in addition to this, it will require the development of the conceptual framework that supports it and the creation of a specific scientific methodology that gives it the characteristic of research in Family Medicine. Conclusion: The family is proposed as the unit of analysis in the scientific research of family medicine and as the main unit of care in the medical care of this specialty.

Keywords: Family medicine. Research. Analysis. Health services.
Correspondencia:

*Enrique Villarreal-Ríos

E-mail: enriquevillarrealrios@gmail.com
Fecha de recepción: 28-06-2021

Fecha de aceptación: 09-10-2021
Disponible en internet: 24-02-2022

Rev Mex Med Fam. 2022;9:31-4 DOI: 10.24875/RMF.21000064

2007-9710 / C 2021 Federación Mexicana de Especialistas y Residentes en Medicina Familiar. Publicado por Permanyer. Este es un artículo open access bajo la licencia CC BY-NC-ND (http://creativecommons.org/licenses/by-nc-nd/4.0/). 


\section{INTRODUCCIÓN}

Cuando se realiza una investigación científica, identificar la unidad de análisis es trascendental, pues es el punto de referencia que define el ámbito de la investigación y la clasifica en un área específica; en torno a la unidad de análisis se construye la propuesta conceptual y metodológica, incluso la descripción e interpretación de los resultados se realiza en torno a ella y aun más, la discusión del documento. Así, por ejemplo, la investigación en pediatría ${ }^{1}$ define como unidad de análisis al infante, lo mismo sucede cuando se investiga en geriatría ${ }^{2}$, en ella el paciente geriátrico es la unidad de análisis, y si se investiga en cirugía ${ }^{3}$, la unidad de análisis es el paciente quirúrgico; no obstante, cuando se realiza investigación en medicina familiar la unidad de análisis se vuelve difusa.

En algunas publicaciones, no obstante, definir a la familia como unidad de análisis, termina siendo la paciente con cáncer de mama ${ }^{4}$, el paciente con trastorno mental ${ }^{5}$ o el paciente con diabetes mellitus ${ }^{6}$; sin embargo, también es verdad que en otras publicaciones se identifica adecuadamente como unidad de análisis a la familia ${ }^{7,8}$ y se abordan temas muy propios de la medicina familiar, entre ellos la dinámica familiar o su funcionalidad $^{9-12}$.

En el protocolo de investigación científica y en el artículo científico de medicina familiar existen puntos críticos que deben revelar el abordaje familiar y a la familia como unidad de análisis, específicamente el marco conceptual y el planteamiento del problema de investigación requiere rebasar el plano individual para llegar al plano grupal, que para el caso específico es la familia.

De igual forma los objetivos y las hipótesis requieren el enfoque familiar: los objetivos, respetando los postulados de cualquier proyecto de investigación, pero redactados intencionalmente en torno a la familia; en las hipótesis estadísticas la información ahí contenida debe reflejar el comportamiento familiar, no el individual, es decir, si se plantea como hipótesis la edad, esta debe corresponder al promedio de edad de los integrantes de la familia y así para cualquier característica familiar que sea motivo de la investigación ${ }^{13}$.

El diseño de la investigación no tiene por qué ser diferente a los diseños ya conoci$\operatorname{dos}^{14-16}$, sin embargo cuando se identifica la población de estudio es menester la definición clara de la población de familias estudiadas, y al señalar la unidad de análisis se deben especificar las características que definen a la familia de estudio; por ejemplo, si se estudia a la familia con embarazo o a la familia con diabetes o a la familia con cardiopatía, se debe ser específico en la definición; una posible descripción es la presencia de al menos uno de los integrantes con el evento de interés (esto lo deberá establecer el investigador en función del marco conceptual y del problema de investigación). Evidentemente, la propuesta se extiende a los grupos de comparación cuando el diseño así lo amerite.

Los criterios de selección se rigen por la misma lógica, se incluye, se excluye o se elimina a una familia, no a un individuo.

Sobra señalar que el cálculo del tamaño de la muestra, independientemente de que se calcule con las mismas fórmulas utilizadas para cada diseño epidemiológico y que se use como referencia el valor expresado en las hipótesis ${ }^{17}$, en el caso de la familia como unidad de análisis se refiere al número de familias que deberán estudiarse; de igual forma, la técnica muestral y el marco muestral tienen por referencia las familias, no los individuos.

Para el caso de la investigación en medicina familiar, las variables evaluadas en la misma escala de medición de cualquier diseño ${ }^{18}$ deben aplicar a todos los miembros de la familia; hacerlo así permite construir la variable del grupo familiar y ser congruente con el abordaje de la investigación. Esto significa que cuando se estudia la escolaridad, debe definirse en función del grado de estudios más alto alcanzado por uno de los integrantes de la familia; 
de igual forma, si la variable es sexo de la familia, se podría decir que existen familias donde predomina el sexo femenino o el masculino, en consecuencia, cuando se reporte el sexo predominante de las familias estudiadas se dirá que predominan las familias con sexo femenino o masculino; evidentemente, el estado civil de la familia está definido por el tipo de relación contractual de pareja que exista entre los integrantes originales.

Derivado de las variables surge el instrumento de recolección o encuesta. Esta tiene un diseño propio en el que se incorpora a todos los integrantes de la familia, y de cada uno de ellos se recolecta información en torno a todas las variables de estudio.

Los aspectos éticos no se encuentran exentos de particularidades. Cuando la unidad de análisis es la familia, específicamente la carta de consentimiento informado ${ }^{19} \mathrm{de}$ be reflejar el conocimiento y autorización de la familia para participar en el estudio, evidentemente existe un representante de la familia que firma a nombre del resto de los miembros, pero la redacción de la carta debe involucrar al grupo familiar.

La redacción de los resultados en el artículo científico ${ }^{20}$ obedece a la misma lógica. En ellos se expresan las características de estudio en función de la familia, en consecuencia, el texto manifiesta el promedio de edad de la familia, el promedio de integrantes de la familia, el sexo predominante en la familia, el tiempo de evolución de ser familia con diabetes o hipertensión o la patología que caracterice a la familia de estudio; de igual forma, los diseños en los que se comparan variables debe reflejar el comportamiento del grupo familiar, no el comportamiento del individuo.

La discusión del artículo científico ${ }^{21}$ no tiene por qué alejarse de este abordaje, en ella la comparación, implicación, explicación, postura del investigador y conclusión se desarrolla en torno al grupo familiar. Si se acepta que lo publicado en la literatura preferentemente involucra al individuo, entonces la discusión de los resultados puede complicarse, pero aun en este escenario el investigador debe forzar la comparación, implicaciones, explicaciones y la postura del investigador para llevar el debate al ámbito familiar y alejarse del individual.

Finalmente, la conclusión, como en cualquier artículo de investigación, debe redactarse en torno al objetivo planteado ${ }^{22}$ y si este se ha propuesto en torno a la familia, la conclusión se redactará también en torno a la familia.

\section{OBJETIVO}

Realizar una propuesta en torno a la familia como unidad de análisis cuando se realiza investigación científica en medicina familiar, para abonar con ello a la discusión del tema.

\section{DISCUSIÓN}

En el contexto de la investigación científica, cuya razón de ser es la generación de conocimiento $^{23}$, lo aquí planteado es una propuesta que tendrá que ser sometida a escrutinio y criticada. Aunado a ello, deberá consolidarse el marco conceptual que la sostenga y de su mano la metodología científica específica que le otorgue la característica de investigación en medicina familiar. Lograr esto significará darle a la medicina familiar el objeto de estudio y la unidad de análisis que le permita distinguirse de la investigación científica en cualquier otra área del conocimiento.

Evidentemente, esta propuesta tendrá detractores y los argumentos esgrimidos serán totalmente validos, seguramente se sostendrá que en medicina familiar ya está perfectamente delimitado el objeto de estudio, en este caso la familia; no obstante, en el ámbito de la investigación y del ejercicio de la práctica clínica, la realidad se aleja un poco de ello. Específicamente, en el caso de la investigación científica en medicina familiar, no obstante presentarse como investigación que estudia a la familia, implícitamente continúa identificando como unidad de análisis al individuo. 


\section{CONCLUSIÓN}

Es verdad que la propuesta se circunscribe al ámbito de la investigación científica, pero implícitamente el abordaje alcanza el modo de estudiar a la familia en la práctica del ejercicio de la especialidad, entender en la investigación científica el estudio de la familia como grupo y no como individuo contribuye a la formación de la cultura organizacional en torno al tema.

\section{FINANCIAMIENTO}

La presente investigación no ha recibido ninguna beca específica de agencias de los sectores públicos, comercial o con ánimo de lucro.

\section{CONFLICTO DE INTERESES}

Los autores declaran no tener conflicto de intereses.

\section{RESPONSABILIDADES ÉTICAS}

Protección de personas y animales. Los autores declaran que para esta investigación no se han realizado experimentos en seres humanos ni en animales.

Confidencialidad de los datos. Los autores declaran que en este artículo no aparecen datos de pacientes.

Derecho a la privacidad y consentimiento informado. Los autores declaran que en este artículo no aparecen datos de pacientes.

\section{BIBLIOGRAFÍA}

1. González-García N, Miranda-Lora AL, Garduño-Espinosa J, Granados-Riverón JT, Méndez-Galván JF, Nieto-Zermeño J, et al. International heterogeneity in coronavirus disease 2019 pediatric mortality rates. Bol Med Hosp Infant Mex. 2021;78(1):24-8.

2. Belmonte Darraz S, González-Roldán AM, Arrebola JM, MontoroAguilar $\mathrm{Cl}$. Impacto del ejercicio físico en variables relacionadas con el bienestar emocional y funcional en adultos mayores. Rev Esp Geriatr Gerontol. 2021;56(3):136-43.

3. Páramo-Zunzunegui J, Alonso-García M, Rodríguez-Villar Drewniak-Jakubowska J, Calvo-Espino P, Cuberes-Montserrat
$\mathrm{R}$, et al. Incidencia de infección quirúrgica y factores de riesgo en cirugía colorrectal. Estudio de cohorte prospectivo. Cir Cir. 2021;89(2):156-62.

4. Acosta-Zapata E, López-Ramón C, Martínez-Cortés ME, ZapataVázquez R. Funcionalidad familiar y estrategias de afrontamiento en pacientes con cáncer de mama. Horiz Sanitario. 2017;16(2):139-48.

5. Casanova-Rodas I, Rascón-Gasca M., Alcántara-Chabelas $H_{\text {, }}$ Soriano-Rodríguez A. Apoyo social y funcionalidad familiar en personas con trastorno mental. Salud Ment. 2015;37(5):443-8.

6. Villarreal-Ríos E, Vargas-Daza ER, Galicia-Rodríguez L, MartínezGonzález L, Neri-Calero C, Hernández-Centeno MG. Costoefectividad de SOHDi en pacientes con diabetes tipo $2 \sin$ hipertensión. Rev Med Inst Mex Seguro Soc. 2010;48(5):535-8.

7. Mendoza-Solís LA, Soler-Huerta E, Sainz-Vázquez L, Gil-Alfaro I, Mendoza Sánchez HF, Pérez-Hernández C. Análisis de la dinámica y funcionalidad familiar en atención primaria. Arch Med Fam. 2006;8(1):27-32.

8. Ordóñez Azuara $Y$, Gutiérrez Herrera RF, Méndez Espinoza $E$ Alvarez Villalobos NA, Lopez Mata D, de la Cruz de la Cruza C. Asociación de tipología familiar y disfuncionalidad en familias con adolescentes de una población mexicana. Aten Primaria. 2020;52(10):680-9.

9. Rangel JL, Valerio L, Patiño J, García M. Funcionalidad familiar en la adolescente embarazada. Rev Fac Med UNAM. 2004;47(1):24-7.

10. Alonso-Castillo MM., Yañez-Lozano Á., Armendáriz-García NA. Funcionalidad familiar y consumo de alcohol en adolescentes de secundaria. Health and Addictions. 2017;17(1):87-96.

11. Sánchez Hidalgo MD. La familia como objeto de investigación: un reto para los profesionales de la salud. Multimed [Internet]. 2016;20(5):4. Disponible en: http://www.revmultimed.sld.cu/index.php/mtm/article/view/382

12. Gutiérrez Herrera RF, Ordóñez Azuara $Y$, Gómez Gómez $C$, Ramírez Aranda JM, Méndez Espinoza E, Martínez Lazcano F. Análisis sobre elementos de contenido en medicina familiar para la identidad profesional del médico familiar. Rev Med La Paz; 2019;25(1):58-67.

13. Icart Isern MT, Canela Soler J. El uso de hipótesis en la investigación científica. Aten Primaria. 1998;21(3):172-8.

14. Fernández E. Estudios epidemiológicos (STROBE). Med Clin 2005;125(Suppl 1):43-8.

15. Schulz KF, Altman DG, Moher D; CONSORT Group. CONSORT 2010 Statement: Updated guidelines for reporting paralle group randomised trials. Trials. 2010;11:32.

16. Donis JH. Tipos de diseños de los estudios clínicos y epidemiológicos. Avan Biomed. 2013;2(2):76-99.

17. Velasco Rodríguez VM, Martínez Ordaz VA, Rois Hernández J, Huazano García F, Nieves Rentería A. Muestreo y tamaño de muestra. México: E-libro.net; 2002.

18. Hernández Ávila M. Epidemiología. Diseño y análisis de estudios. México: Editorial Médica Panamericana; 2007.

19. Comisión Nacional de Bioética. Comités de Ética en Investigación [Internet]. Gobierno de México, Comisión Nacional de Bioética; 2021. Disponible en: https://www.gob.mx/salud/conbioetica/ es/articulos/comites-de-bioetica?idiom=es

20. Revista Mexicana de Medicina Familiar. Instrucciones para autores [Internet]. México: Revista Mexicana de Medicina Familiar. Disponible en: http://www.revmexmedicinafamiliar.org/authors-instructions.php

21. Medicina de Familia. SEMERGEN. Guía para autores [Internet] Elsevier, Medicina de Familia. SEMERGEN. Disponible en: https:// www.elsevier.es/es-revista-medicina-familia-semergen-40-normas-publicacion

22. Evans-Meza R, Galan-Rodas E. Redacción del artículo científico en medicina. Rev Hisp Cienc Salud. 2017;3(1):23-8.

23. Bunge M. La ciencia, su método y su filosofía [Internet] Disponible en: http://www.posgrado.unam.mx/musica/lecturas/LecturalntroduccionInvestigacionMusical/epistemologia/ Mario-Bunge-la-Ciencia-su-Metodo-y-Filosofia.pdf 